\title{
Incidental Findings in Youths Volunteering for Brain MRI Research
}

\author{
R.E. Gur, D. Kaltman, E.R. Melhem, K. Ruparel, K. Prabhakaran, M. Riley, E. Yodh, H. Hakonarson, T. Satterthwaite, and R.C. Gur
} \& O. ${ }_{2}$

\begin{abstract}
BACKGROUND AND PURPOSE: MRIs are obtained in research in healthy and clinical populations, and incidental findings have been reported. Most studies have examined adults with variability in parameters of image acquisition and clinical measures available. We conducted a prospective study of youths and documented the frequency and concomitants of incidental findings.
\end{abstract}

MATERIALS AND METHODS: Youths $(n=1400)$ with an age range from $8-23$ years were imaged on the same 3T scanner, with a standard acquisition protocol providing $1.0 \mathrm{~mm}^{3}$ isotropic resolution of anatomic scans. All scans were reviewed by an experienced board-certified neuroradiologist and were categorized into 3 groups: 1) normal: no incidental findings; 2) coincidental: incidental finding(s) were noted, further reviewed with an experienced pediatric neuroradiologist, but were of no clinical significance; 3) incidental findings that on further review were considered to have potential clinical significance and participants were referred for appropriate clinical follow-up.

RESULTS: Overall, 148 incidental findings (10.6\% of sample) were noted, and of these, 12 required clinical follow-up. Incidental findings were not related to age. However, whites had a higher incidence of pineal cysts, and males had a higher incidence of cavum septum pellucidum, which was associated with psychosis-related symptoms.

CONCLUSIONS: Incidental findings, moderated by race and sex, occur in approximately one-tenth of participants volunteering for pediatric research, with few requiring follow-up. The incidence supports a 2-tiered approach of neuroradiologic reading and clinical input to determine the potential significance of incidental findings detected on research MR imaging scans.

$\mathbf{T}$ he prevalence of incidental findings in nondiagnostic brain MR imaging research has been examined in empirical studies, case reports, reviews, and ethical commentaries. ${ }^{1-3}$ Empirical studies have reported the prevalence of incidental findings in various samples commonly including at least 100 subjects. ${ }^{4}$ Of approximately 35 such studies, $75 \%$ evaluated adults, $10 \%$ were conducted in autopsies, and a few in pediatric populations. Of those, even fewer applied high-field (3T) high-resolution sequences with $1.0-\mathrm{mm}^{3}$ isotropic or smaller voxels, which may result in more incidental findings. Given the increasingly common use of high-resolution brain MR imaging in pediatric pop-

Received December 12, 2012; accepted after revision January 3, 2013.

From the Brain Behavior Laboratory (R.E.G., D.K., K.R., K.P., M.R., E.Y., T.S., R.C.G.), Department of Psychiatry, and Division of Neuroradiology (R.E.G., E.R.M., R.C.G.), Department of Radiology, Perlman School of Medicine, University of Pennsylvania, Philadelphia; and Center for Applied Genomics (H.H.), Children's Hospital of Philadelphia, University of Pennsylvania, Philadelphia, Pennsylvania.

Please address correspondence to Raquel E. Gur, MD, PhD, Neuropsychiatry, 10 Gates, 3400 Spruce, Philadelphia, PA 19104; e-mail: Raquel@upenn.edu

- Indicates open access to non-subscribers at www.ajnr.org

Evidence-Based Medicine Level 2

http://dx.doi.org/10.3174/ajnr.A3525 ulations, establishing both a baseline rate for incidental findings and a framework for evaluation is of importance.

Prior work provides data regarding a possible range of rates of incidental findings in heterogeneous populations, commonly adults. The first large-scale study of 1000 asymptomatic volunteers (age range, 3-83 years) reported an incidental findings prevalence of $4.8 \%{ }^{5}$ Most studies reported rates excluding sinus disease. Kim et al, ${ }^{1}$ in a study of 225 neurologically healthy subjects (age, $0-18$ years), established an incidental findings prevalence of approximately $11 \%$, and males had twice the occurrence of incidental findings as females. Seki et $\mathrm{al}^{6}$ evaluated 110 healthy children (age, 5-8 years) in a single scanner (1.5T) with the same imaging sequence and found $11 \%$ with incidental findings. A meta-analysis of 16 studies including nearly 20,000 scans, mainly in adults, reported inconsistent incidental findings and found that the rate of incidental findings was related to image resolution. ${ }^{4}$ With sequences considered low-resolution by present standards, incidental findings prevalence was approximately $3-4 \%$. Thus, relatively few studies have examined incidental findings in youths, and prior studies have been limited by both the effective resolution of the imaging sequence used and small sample size. 
Clinical correlates of incidental findings have received some attention because incidental findings may be indicative of underlying pathology. For example, children with developmental delay of unknown etiology, according to retrospective chart reviews, were more likely ( 1.8 odds ratio) to have incidental findings than healthy control subjects. ${ }^{7}$ In a recent meta-analysis, Trzesniak et $\mathrm{al}^{8}$ concluded that the presence of a large cavum septum pellucidum is associated with schizophrenia spectrum disorders. However, no study has examined whether a finding of cavum septum pellucidum in an epidemiologically accrued sample is associated with increased incidence of psychotic features.

The Philadelphia Neurodevelopment Cohort provides an opportunity to evaluate incidental findings in a population-based sample of 1400 young people, ages $8-21$ years. This is the largest single-site, single-scanner, high-resolution pediatric MR imaging study with a similar representation of whites and African Americans. We conducted a prospective examination of incidental findings yielding the largest standardized sample to date of high-resolution brain MRI in pediatric research. Our goal was to determine the prevalence of incidental findings in this young population and to examine whether certain findings were associated with demographic characteristics or correlated with clinical measures.

\section{MATERIALS AND METHODS \\ Subjects}

Subjects were recruited as part of the Philadelphia Neurodevelopment Cohort, a collaborative National Institute of Mental Health-funded Grand Opportunity study between the Brain Behavior Laboratory at the University of Pennsylvania and the Center for Applied Genomics at Children's Hospital of Philadelphia. The overall goal of the project is to examine a large sample of genotyped youths, ascertained through the Children's Hospital of Philadelphia network, who provided consent/assent to be re-contacted for the study of complex pediatric disorders. The Children's Hospital of Philadelphia network includes a mixture of urban and suburban clinics as well as a tertiary care hospital. Therefore, the sample is diverse and represents the range of health conditions found in youths. Inclusion criteria were age 8-21 years; general good health, as indicated by review of electronic medical records and telephone screen; the ability to provide signed informed consent or assent; English proficiency; and physical and cognitive capability to participate in a comprehensive assessment. More than 9000 eligible participants who met inclusion criteria underwent further direct neuropsychiatric assessment and computerized neurocognitive testing. ${ }^{9}$ The neuropsychiatric assessment consisted of evaluation of medical, neurologic, and psychiatric disorders, ${ }^{10}$ including psychotic symptoms. ${ }^{11}$ The computerized neurocognitive battery included measures of executive functions, memory, reasoning, social cognition, and sensorimotor speed, as detailed by Gur et al. ${ }^{12}$

A randomly selected subsample of 1400 subjects, stratified by age and sex and meeting criteria for MR imaging compatibility, completed the MR imaging study and were included in the present analysis. ${ }^{13}$ Notably, subjects were not recruited on the basis of any pre-existing disorder or symptoms. There were 670 males (48\%) and 730 females (52\%); 627 whites (45\%), 589 African
Table 1: Sample demographics by group

\begin{tabular}{lcc}
$\begin{array}{c}\text { Participant } \\
\text { Characteristic }\end{array}$ & $\begin{array}{c}\text { Normal Finding } \\
\text { Group }(\boldsymbol{n}=\mathbf{1 2 5 2})\end{array}$ & $\begin{array}{c}\text { Incidental Finding } \\
\text { Group ( } \boldsymbol{n}=\mathbf{1 4 8})\end{array}$ \\
\hline Age, y & $14.72 \pm 3.59$ & $14.85 \pm 3.92$ \\
Education, y & $7.64 \pm 3.50$ & $7.81 \pm 3.77$ \\
Sex, \% & & \\
$\quad$ Male & 48 & 49 \\
Female & 52 & 51 \\
Race, \% & & 49 \\
White & 45 & 40 \\
African American & 42 & 11 \\
Other & 13 & \\
\hline
\end{tabular}

Americans (42\%), and $184(13 \%)$ subjects of other or mixed races. The age distribution is presented in Table 1.

\section{MR Imaging Acquisition}

All MRIs were acquired on the same 3T scanner (TIM Trio; Siemens, Erlangen, Germany), with a 32-channel head coil, located in the Department of Radiology at the Hospital of the University of Pennsylvania. All scans were conducted by a board-certified MR imaging technologist by use of a standard protocol. Foam cushions were used to stabilize the head; no sedation was used. The sequence used was an axial T1-weighted magnetization-prepared rapid acquisition of gradient echo, with parameters TR/TE, 1810/3.51 ms; TI, $1100 \mathrm{~ms}$; FOV, $180 \times 240 \mathrm{~mm}$; effective voxel resolution, $1 \times 1 \times 1 \mathrm{~mm}^{3}$; scanning time, 3 minutes, 28 seconds. Before the study proper, participants were acclimated to the MR imaging environment by use of a mock scanning session in a decommissioned MR imaging scanner.

\section{Classification of Findings}

All scans were reviewed in the neuroradiology reading room at the Hospital of the University of Pennsylvania according to standard clinical procedures. Twenty board-certified neuroradiologists read the images and provided a written clinical report. Five of them reviewed $60 \%$ of the scans. The scans and reports were electronically transferred to a data base, and one investigator (R.E.G.) reviewed all reports and classified each as normal or coincidental:

Normal. No incidental finding noted.

Coincidental. All incidental finding(s) that were noted in the clinical report were further reviewed by an experienced pediatric neuroradiologist (E.R.M.) to determine their clinical pertinence. Findings that were not clinically significant were not communicated to the subject (nor to their parent/guardian, if applicable).

Follow-up. Coincidental findings of potential clinical significance were communicated to the subjects and/or their parent or guardian, with appropriate referrals as indicated.

\section{Incidental Finding Categorization}

As in previous studies, 6 categories were created to further analyze common incidental findings: 1) pineal cyst, 2) other cyst, 3) cavum septum pellucidum, 4) other ventricular abnormalities (prominence or asymmetry of ventricle or CSF), 5) vascular abnormalities (asymmetric prominence of artery or vein, developmental venous anomaly, tortuous artery, possible infarct, possible aneurysm), and 6) cerebellar abnormalities (cerebellar tonsillar 


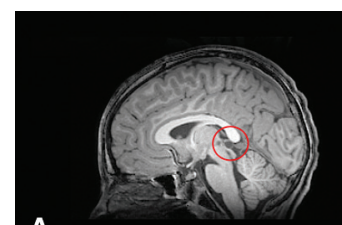

A
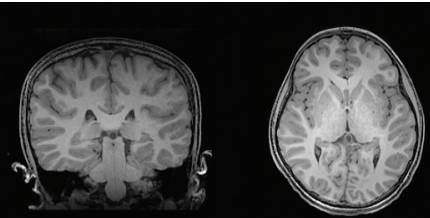

(1)

B
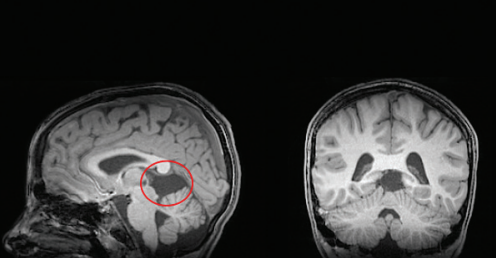

(e)

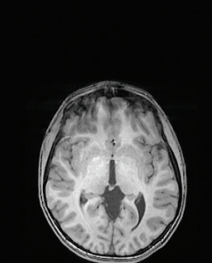

C
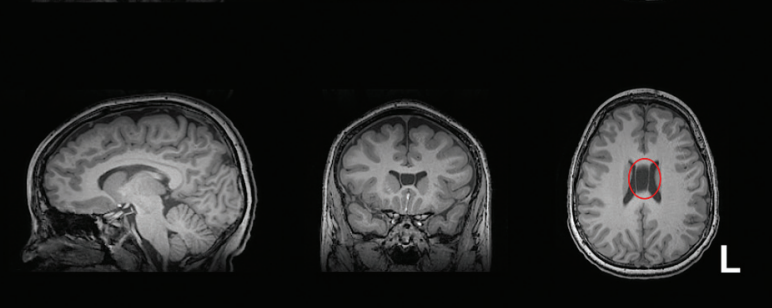

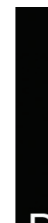

$\mathrm{D}$
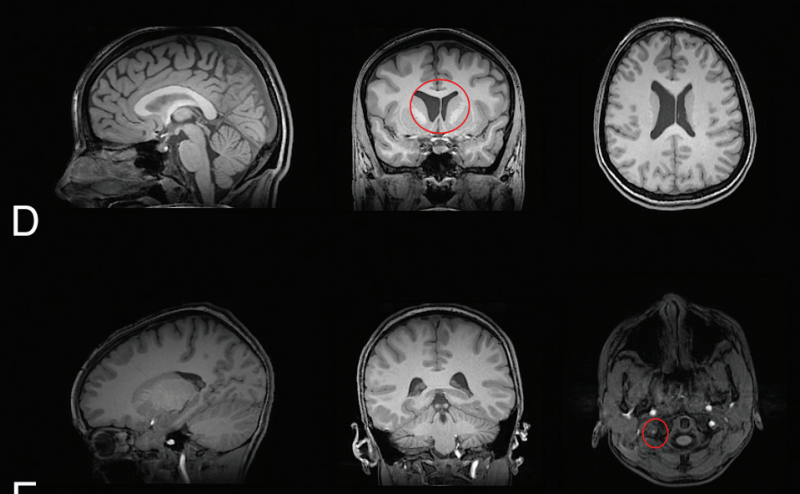

E

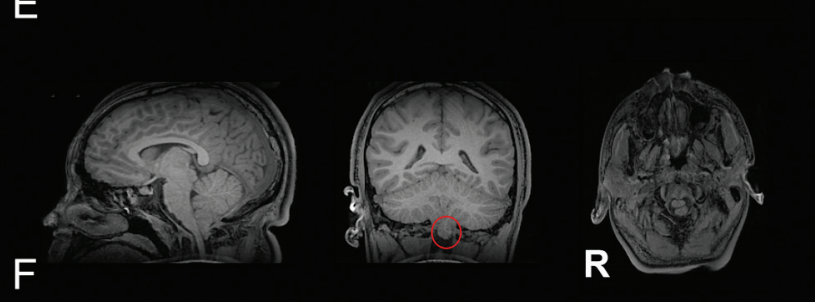

FIG 1. Examples of incidental findings categories. $A$, Pineal cyst; $B$, other cysts; $C$, cavum septum pellucidum; $D$, ventricular abnormalities; $E$, vascular abnormalities; $F$, cerebellar abnormalities.

ectopia, Chiari I malformation, prominent retrocerebellar space, low-lying cerebellar tonsils, mega cisterna magna, cerebellar cyst). Examples for these categories are provided in Fig $1 A-F$.

Two specific findings were excluded from the present analysis. As in prior work, findings outside the cranial vault were not considered in this report. This includes nasopharynx and sinus conditions, such as opacification of the sinus or sinus mucosal disease. Findings of dilated perivascular spaces were excluded from analysis because of inconsistent reporting.

\section{Statistical Data Analysis}

Statistical analysis comparing the demographic characteristics between groups was performed by use of the Student $t$ test for age and the $\chi^{2}$ test for proportions (sex and race). For each incidental finding category, logistic regression was performed to determine whether demographic characteristics (age, sex, race) predicted the odds of the incidental finding. Interactions within demographic variables were studied, but nonsignificant interactions were not included in the final models. All models were evaluated for lack of fit with the Hosmer-Lemeshow test. Analyses were implemented by use of R software (version 2.15.0, http://www.r-project.org/). ${ }^{14}$

To test the hypothesis of increased psychotic features in patients with cavum septum pellucidum, ${ }^{8}$ we compared individuals with this incidental finding with participants from the larger sample who matched most closely on age, sex, and race. Specifically, each subject was paired with all participants in the sample who had the same values on these demographics. The means of the 2 groups on the psychosis score ${ }^{11}$ were compared by means of a Student $t$ test. This part of the analysis was implemented in SAS, version 9.2 (SAS Institute, Cary, North Carolina). ${ }^{15}$

\section{RESULTS}

\section{Incidental Findings}

Of the 1400 scans, 1252 were read as normal (89.42\%). There were 148 scans with incidental findings $(10.57 \%)$, and of these, 12 in-
Table 2: Frequency of incidental findings

\begin{tabular}{lccl}
\hline \multicolumn{1}{c}{ Finding } & Count & Prevalence & Literature \\
\hline Pineal cyst & 34 & $2.43 \%$ & $1-10 \%$ \\
Other cyst & 19 & $1.36 \%$ & $1-15 \%$ \\
Cavum septum pellucidum & 16 & $1.14 \%$ & $1-20+\%$ \\
Other CSF abnormalities & 33 & $2.36 \%$ & N/A \\
Vascular abnormalities & 36 & $2.57 \%$ & N/A \\
Cerebellar abnormalities & 33 & $2.36 \%$ & $1-3 \%$ ectopia \\
Coincidental & 136 & $9.71 \%$ & $2-20 \%$ \\
All incidental & 148 & $10.57 \%$ & $3-85 \%$ \\
\hline
\end{tabular}

The literature values are mostly from adults. Counts do not add to 148 because some subjects have more than 1 finding.

dicated clinical follow-up. The age distribution of subjects with any incidental findings did not differ from those without incidental findings $(P=.69)$. The sex distribution of those with incidental findings likewise did not differ from that of the total sample $(P=.52)$, nor did racial distribution $(P=.41)$. Table 2 shows the count and prevalence for each incidental finding category.

\section{Association with Specific Incidental Findings}

Multiple logistic regression analyses revealed several significant relationships between demographic indicators and the existence of a specific incidental finding. When controlling for age and sex in the model, whites were 195\% more likely than African Americans to have a pineal cyst $(\mathrm{OR}=2.95, \mathrm{CI}=1.20-7.22, P=.02)$; females were $76 \%$ less likely than males to have a cavum septum pellucidum $(\mathrm{OR}=0.24, \mathrm{CI}=0.06-0.92, P=.04)$.

There was a significant age by race interaction for cerebellar abnormality $(P=.02)$ and ventricular abnormality $(P=.04)$. When controlling for sex, whites were more likely to have cerebellar abnormality than African Americans, and this effect of race on the odds of having cerebellar abnormality decreased with age. When controlling for sex, whites were less likely to have ventricular abnormality than African Americans, and this effect of race likewise decreased with age. All final multivariable adjusted mod- 
els were reliable $(P>.2)$ on the basis of the Hosmer-Lemeshow goodness-of-fit test.

Notably, the 16 cases with cavum septum pellucidum endorsed more psychotic symptoms than those with no incidental findings, matched for age and $\operatorname{sex}(n=459, t=7.20, d f=471, P<$ .0001).

\section{DISCUSSION}

In this prospective analysis of incidental findings in brain MR imaging of presumed healthy subjects, we established that incidental findings are common, with a prevalence of $>10 \%$. Although the prevalence of such findings varies widely in the literature, from as low as $3 \%$ to $>80 \%$, the meta-analysis by Morris et $\mathrm{al}^{4}$ reported a prevalence of approximately $4 \%$. This value reflected a combination of studies with an effective voxel resolution of at best $2 \times 2 \times 2 \mathrm{~mm}^{3}$ but typically $3 \times 3 \times 3 \mathrm{~mm}^{3}$; this is referred to as "high resolution" but is in fact coarse by current standards. Despite our exclusion of sinus-related findings, which some studies in the meta-analysis included, we obtained a value more than twice as large as the Morris et al value.

There are several possible reasons for this finding. First and foremost, our study used a higher-field magnet (3T) and acquired higher-resolution (1- $\mathrm{mm}^{3}$ isotropic voxels) images than any data set included in the Morris et $\mathrm{al}^{4}$ meta-analysis. This enhanced resolution probably allowed greater sensitivity to detect small cysts and vascular anomalies. In addition, the resolution and the sequence used allowed for a larger field of view than that used in many previous analyses of incidental findings.

This report represents the largest study to date of incidental findings in an adolescent population. Although no significant correlations were detected between age and the prevalence of incidental finding, the narrow age band under investigation limited our ability to detect any such differences; subjects were divided into age bins of only 2 years for this analysis, compared with the $>10$-year age bins used in studies with more heterogeneous samples.

Analysis of specific incidental findings with logistic regression revealed several novel findings. Whites were more than twice as likely as African Americans to have any cyst and nearly 3 times more likely to have a pineal cyst. In addition, whites were more likely to have cerebellar findings, whereas African Americans had a greater incidence of ventricular findings. These latter effects became weaker with age. Earlier studies have been limited in their ability to examine the effects of race by smaller and more homogeneous samples.

Notably, males were approximately 5 times more likely than females to have a cavum septum pellucidum. Several studies suggest that the presence of a cavum septum pellucidum is significantly correlated with psychotic symptoms, also more prevalent in males. Whereas a recent meta-analysis by Trzesniak et $\mathrm{al}^{8}$ suggested that only a "large" cavum was associated with schizophrenia spectrum disorders, in our sample we found significantly greater incidence of reporting psychotic symptoms in the subsample with cavum septum pellucidum. However, the small sample size precludes establishing a correlation between symptom severity and septum size. Longitudinal studies are needed to ex- amine whether individuals with cavum septum pellucidum are more likely to have psychotic illness.

The findings of this study can be used to better inform research participants about the potential risks and benefits of volunteering. As previously suggested, ${ }^{1}$ further research on the prevalence of incidental findings could inform the risk/benefit calculus of informed consent for research participants if the anticipated prevalence of a finding becomes well established. The present study provides a highly standardized description of the prevalence of incidental findings in pediatric neuroimaging research at a resolution and magnetic field strength widely used by researchers today. Although ethics was not the primary focus of this study, our approach to handling incidental findings was commensurate with that recommended by bioethicists. ${ }^{16-18}$ Expert review of the research-grade images was performed routinely, with further review to determine the clinical significance. Despite an overall prevalence of roughly $11 \%$, only 12 subjects were actually contacted for follow-up after expert consultation. The lack of involvement of a neuroradiology expert could cause unnecessary over-reporting of findings to research subjects.

Our study has several limitations. Although subjects were not recruited on the basis of any pre-existing condition, they were recruited through a children's hospital network, and most were from the greater Philadelphia area. To compensate for the regional bias, we accounted for race, sex, and age in our model. All 3 factors were approximately balanced in the overall sample. In addition, several neuroradiologists conducted the initial image readings. Although standardized criteria are used to identify the various findings analyzed in this study, we cannot discount the potential variability in selective reporting of certain findings. To address this issue, a single investigator (R.E.G.) reviewed all of the neuroradiology reports and a single pediatric neuroradiologist (E.R.M.) reviewed all incidental findings. The 6 incidental finding categories were not determined a priori but were instead generated on the basis of their prevalence and are consistent with other reports.

Notwithstanding these limitations, our large-scale, prospective study has identified several common incidental findings in a pediatric population. A single high-field scanner and high-resolution imaging allow this study to establish a benchmark for the prevalence of incidental findings in an ethnically diverse population of male and female youths. The substantial prevalence of incidental findings indicates the utility of including a clinical reading of MR imaging scans performed on research participants. On the other hand, the small number of findings requiring follow-up support the use of further expert review so that participants are not unnecessarily alarmed. Our preliminary finding of increased reporting on psychotic symptoms in a subgroup of youths with cavum septum pellucidum supports longitudinal research on this group.

\section{CONCLUSIONS}

Our large-scale, population-based pediatric study with the use of high-resolution neuroimaging found a prevalence of approximately $10 \%$ incidental findings. This number is higher than the limited pediatric literature and supports the value of clinical reading of MR imaging performed as part of research studies. Notably, 
some findings were moderated by race, with pineal cysts and cerebellar incidents more evident, whereas ventricular findings were less frequent in whites than in African Americans.

\section{ACKNOWLEDGMENTS}

This research was supported by National Institutes of Health grant MH089983. We thank the Division of Neuroradiology; MR imaging technologists Norman Butler, Doris Cain, and Tanya Kurtz; the Brain Behavior Laboratory MR imaging acquisition team, including Jeffrey Valdez, Ryan Hopson, Nicholas DeLeo, and Jack Keefe; and the participants and families.

Disclosures: Raquel Gur_RELATED: Grant: NIH/NIMH, ${ }^{\star}$ Comments: The work was supported by federal research grants acknowledged in the manuscript. David Kaltman-RELATED: Grant: National Institute of Mental Health.* Karthik Prabhakaran-RELATED: Grant: National Institute of Mental Health.* Marisa RileyRELATED: Grant: NIH/NIMH.* Elliott Yodh—RELATED: Grant: NIH/NIMH.* Hakon Hakonarson-RELATED: Grant: NIMH, ${ }^{\star}$ Comments: Support from NIMH to study Neurodevelopmental Genomics: Trajectories of Complex Phenotypes (1RC2MH089924-01). Theodore Satterthwaite-RELATED: Grant: $\mathrm{NIMH},{ }^{*} \mathrm{Com}-$ ments: MH089983, MH089924; UNRELATED: Grants/Grants Pending: NIMH,* APIRE,* NARSAD.* Ruben Gur-RELATED: Grant: NIMH;* Support for Travel to Meetings for the Study or Other Purposes: NIMH; UNRELATED: Expert Testimony: Courts and Federal Defenders, Comments: I have provided medical-legal expert testimony, mostly in capital cases, none related specifically to this manuscript or work; Grants/Grants Pending: NIMH* (*money paid to institution).

\section{REFERENCES}

1. Kim BS, Illes J, Kaplan RT, et al. Incidental findings on pediatric MR images of the brain. AJNR Am J Neuroradiol 2002;23:1674-77

2. Weber $F$, Knopf $H$. Incidental findings in magnetic resonance imaging of the brains of healthy young men. J Neuro Sci 2006;240:81-84

3. Vernooij MW, Ikram MA, Tanghe HL, et al. Incidental findings on brain MRI in the general population. $N$ Engl $\mathrm{J} \mathrm{Med}$ 2007;357:1821-28

4. Morris Z, Whiteley WN, Longstreth WT Jr, et al. Incidental findings on brain magnetic resonance imaging: systematic review and metaanalysis. BMJ 2009;339:b3016

5. Katzman GL, Dagher AP, Patronas NJ. Incidental findings on brain magnetic resonance imaging from 1000 asymptomatic volunteers. JAMA 1999;282:36-49

6. Seki A, Uchiyama H, Fukushi T, et al; Japan Children's Study Group.
Incidental findings of brain magnetic resonance imaging study in a pediatric cohort in Japan and recommendation for a model management protocol. J Epidemiol 2010;20(Suppl 2):S498-504

7. Gupta S, Kanamalla U, Gupta V. Are incidental findings on brain magnetic resonance images in children merely incidental? J Child Neurol 2010;25:1511-16

8. Trzesniak C, Oliveira IR, Kempton MJ, et al. Are cavum septum pellucidum abnormalities more common in schizophrenia spectrum disorders? A systematic review and meta-analysis. Schizophr Res 2011;125:1-12

9. Gur RC, Richard J, Calkins ME, et al. Age group and sex differences in performance on a computerized neurocognitive battery in children age 8-21. Neuropsychology 2012;26:251-65

10. Kaufman J, Birmaher B, Brent D, et al. Schedule for Affective Disorders and Schizophrenia for School-Age Children-Present and Lifetime Version (K-SADS-PL): initial reliability and validity data. JAm Acad Child Adolesc Psychiatry 1997;36:980-88

11. Miller TJ, McGlashan TH, Rosen JL, et al. Prodromal assessment with the structured interview for prodromal syndromes and the scale of prodromal symptoms: predictive validity, interrater reliability, and training to reliability. Schizophr Bull 2003;29:703-15

12. Gur RC, Richard J, Hughett P, et al. A cognitive neuroscience-based computerized battery for efficient measurement of individual differences: standardization and initial construct validation. $J \mathrm{Neu}$ rosci Methods 2010;187:254-62

13. Satterthwaite TD, Wolf DH, Loughead J, et al. Impact of in-scanner head motion on multiple measures of functional connectivity: relevance for studies of neurodevelopment in youth. Neuroimage 2012;60:623-32

14. R Development Core Team. R: a language and environment for statistical computing. R version 2.12.0. Available at: http://www.R-project.org. Accessed October 15, 2010

15. SAS software, Version 9.2 of the SAS System for Linux, SAS Institute Inc, Cary, North Carolina; 2009

16. Illes J, Chin VN. Bridging philosophical and practical implications of incidental findings in brain research. J Law Med Ethics 2008;36:298-304

17. Wolf SM, Lawrenz FP, Nelson CA, et al. Managing incidental findings in human subjects research: analysis and recommendations. $J$ Law Med Ethics 2008;36:219-48

18. Kumra S, Ashtari M, Anderson B, et al. Ethical and practical considerations in the management of incidental findings in pediatric MRI studies. J Am Acad Child Adolesc Psychiatry 2006;45:1000-06 